\title{
TEST METHOD FOR RHEOLOGICAL BEHAVIOR OF MORTAR FOR BUILDING WORK
}

\author{
Bogdan KOROBKO*, levgen VASYLIEV
}

\author{
*Electromechanic Faculty, Department of Construction Machinery and Equipment, Poltava National Technical Yuri Kondratyuk University, \\ Poltava, Pershotravnevyi Avenue 24, Ukraine \\ bogdan.korobko@ukr.net, vas.eugene@gmail.com
}

received 25 August 2016, revised 18 July 2017, accepted 7 August 2017

\begin{abstract}
This paper offers a test method for rheological behavior of mortars with different mobility and different composition, which are used for execution of construction work. This method is based on investigation of the interaction between the valve ball and the mortar under study and allows quick defining of experimental variables for any composition of building mortars. Certain rheological behavior will permit to calculate the design parameters of machines for specific conditions of work performance - mixing (pre-operation), pressure generation, pumping to the work site, workpiece surfacing.
\end{abstract}

Key words: Mortar Mixture, Rheology, Mobility Of Mortar, Pumping Over, Mixing

\section{INTRODUCTION}

Adoption of innovative mechanised technologies of finishing works execution broadens application of hauling equipment for mortar mixtures and tender mixes pipelining. For this purpose, the usage of highly productive short-duration pulse mortar pumps provides an opportunity to mechanize not only the supply of constructional materials to working places, but also to execute the main processing steps: mechanized plastering and decorative coating in the process of finishing the walls; laying mortar mixtures when sealing structural joints; mixture placing on the floor, dampproofing.

Best practices in operating mortar pumps when phasing in mechanized technology points to further improvement of these machinery through their reliability growth and by reducing energy costs for repumping at the cost of flow fluctuation decrease and volumetric losses in the fluid end of the mortar pump (Korobko and Vasyliev, 2012).

User experience of mortar pumps lends credence to the fact, that the level of mortar mixtures' volumetric losses during repumping is pretty much conditioned upon the interaction between the operating environment and mortar pump's working members, that are defined by their elastic characteristics. However, the deficiency of quantitative characteristics of elastic characteristics of mortar mixtures with different composition and mobility adds complexity to the development of effective ways of improving volume efficiency of the created new-generation mortar pumps. That's why theoretical studies and field researches of mortar mixtures' elastic characteristics are sorely needed for the improvement of the ways of mortar mixtures' pipelining.

The major complications in the process of repumping mortar mixtures are caused by their rheological characteristics. The mortar mixture is structured thixotropic liquid, the main rheological characteristics of which are structured viscosity and critical shear stress. When lowering the mobility of the mortar to the technologically required magnitude $(10 \ldots 8 \mathrm{~cm})$, these figures assume the values, where the advance of mortar mixture flow over the pipeline evokes rather strong resistance. Under such conditions the process of filling the processing chamber with mortar mixture is made difficult under the influence of pressure decrease from the level $0.1 \mathrm{MPa}$. Service conditions of valve operation also decline, what causes the delay of the valve closure and sometimes onstate "hanging" and dramatic reduction of pump volumetric efficiency (Korobko, 2016).

With allowance for this, taking into account the abovesaid peculiarities when developing and estimating service properties of hauling equipment is an important task.

Construction mix as a service which is piped through pipelines, has a set of features that must be taken into account in the design of mortar pumps, as well in their operation.

Mobility of construction mixtures can be increased by adding fluidifiers into them. Highly mobile mixtures with the mobility of 11-12 cm are used for spraying the first coat when plastering surfaces in order to increase the cohesive resistance of the plastering coat with the brick masonry. Mixtures with mobility of 8-9 cm are transferred through pipelines with difficulty; they are hardly sucked into the sucking working chamber of the mortar pump; valve balls may "hover" in them, what may cause failure in the operation of mortar pumps. However, these mixtures allow to lay on maximum-deep plaster layers during the minimum amount of passes; furthermore, they bring less moisture into building constructions.

The main processing characteristics of plasters:

- easy laying on, which is characterized by the mobility of construction mixture, in other words - by the ability to flow under the influence of its own weight or external forces;

- water fixation - the property to retain excess water in the process of suction, thereby there are no water losses when laying mortar mixture on the porous soil, as well as it unscrambles insignificantly during the transportation of mortars;

- compressive breaking strength of the mortar stone; 
- freeze-thaw durability, which is determined by the ability of mortar mixture sample to withstand in a water-saturated state of a certain number of cycles of alternate freezing and thawing.

The slurry formulation comprises a significant number (up to $70 \%$ of volume) of dispersed hard phase. But such state of solution is not stable. If the size or the direction of speed changes due to the hard phase immiscibility or aggregation, there arise deposits of solid particles, which increase the resistance to flow advance and seal paravalvular blowholes. In addition, if the pump stops for extended lengths of time, except immiscibility, partial hardening of the pumped medium takes place, especially on hydraulic binding agents. Consequently, routine breaks in the work of pump require timeous flushing of cavities in the processing chamber and pipelines.

Density composition of solids drastically worsens operation conditions of friction couples in the fluid end of the mortar pump seals, muff, piston and rod. Another disadvantage, that accelerates rubbing parts' wear, is the presence in the solution of water, which in combination with chemically-active cementing phases causes corrosive action, in other words, it conditions erosion corrosion and particulate erosion of the mortar pump's parts.

Against this background, an important task is to take into account the mentioned peculiarities in estimating and developing the service properties of hauling equipment.

When analyzing the influence of solution properties on the pumping process, it is necessary to use their flow factor, which is in the range of $5 \ldots 12 \mathrm{~cm}$ and characterizes the convenience of transportation and mortar laying on workpiece surfaces. However, this characteristic does not reveal the interaction between the solution and pump components and can describe this process only when building empirical statistic dependences.

In this regard, when developing new samples of mortar pumps, it seems essential and important today to analyze the method of scoring the properties of solutions in order to reveal the mechanism of manifestation of these properties in the process of conveyance by the mortar pump.

Rheological properties of solutions are determined by using the viscometres of rotatory operation. The principle of operation of these devices is based on the studying of relative rotational motion of cylinders, between which the test medium is situated. The usage of this method for building mortars is limited by the slot width between the cylinders and is efficient only for very workable plasters. The value of structured viscosity and shift voltage depend mainly on the flow rate (the intensity of structural links destruction). In different sources the represented data on the significance of the pointed out rheological properties have quite a broad band, as they were received in different velocity (kinematic) conditions. In this regard, when developing a machine with specific operating factors, it is necessary to do measuring operations on the used solution in order to correct real value of thresholds, which characterize rheological properties of the service (medium).

The required properties of the mortar are achieved by cementaggregate compatibility, namely by the amount of material by weight or volume, which falls per $1 \mathrm{~m} 3$ of mortar mixture, or by the ratio of each mortar's component to the binder also by mass or volume, at that the binder discharge is taken as a unit (Chen et al., 2013; Kheradmand M. et al., 2014).

It should be noted that during pipeline transportation it is necessary to provide plasticity of mortar mixture and appropriate convenience of pumping under pressure. The latter is peculiar to such mixtures, that during transportation by the mortar pump do not form stagnation zones or sand seals in pressure hoses, and are pumped as a continuous flow. These showings are determined by the mobility of the mixture and they are provided by adjusting to the required condition in the mixer of the plastering machine (Kim K.H. et al., 2012).

Further improvement of the facilities for mortar mixture supply through pipelines and the development of rational parameters of the structural components in the fluid end of the mortar pump with minimal resistance to pumping under pressure require studying the interaction of the working body and the valve assemblies with the flow of the pumped medium. In order to get that done, again, it is necessary to define rheological characteristics of the pumped mortar mixture.

\section{MEASURING TECHNIQUE FOR RHEOLOGICAL PROPERTIES OF MORTAR MIXTURES}

Flow behavior of mortar mixtures, which belong to structural fluids (Almeida and Sichieri, 2007; Binici et al., 2012; GonzálezFonteboa and Martínez-Abella, 2008), can be characterized to the fullest extent possible by the formula of Shvedov-Bingham

$\tau=\tau_{0}+\eta \cdot \frac{d V}{d n}$

where: $\tau$ - is the shear stress, $\mathrm{Pa} ; \tau_{0}$ - is the critical shear stress, after the achievement of which the liquid comes into motion, $\mathrm{Pa}$; $\eta-$ is the absolute viscosity coefficient Pa.s; $\frac{\mathrm{dV}}{\mathrm{dn}}-$ is the velocity gradient in the cross flow direction.

The critical shear stress $\tau_{0}$ and the absolute viscosity coefficient $\eta$ are the main rheological properties of the service under consideration and determine the nature of the interaction between mortar mixture flow with the elements of the fluid end of the mortar pump.

The value of the indicated parameters varies over the residence time of the static fluid (at zero speed) and depends on a number of factors (Cuia et al., 2015). This is, in particular, the ratio of the mortar mixture ingredients, the quantity of water, which is comprised into the mortar mixture, the flow rate of speed. In addition, rheological characteristics depend on specific measurement conditions (Perrot et al., 2013).

This explains the wide disagreement in the values of the mentioned parameters $\tau_{0}$ and $\eta$, given in different literature sources (Assaad and Daou, 2014; Reis et al., 2011). Therefore, in the process of pumping the mortar mixture, it is necessary to define the indicated parameters for mortar mixtures with fixed composition and specified mobility.

In view of this, the usage of traditional viscosity measurement devices is obstructed, as far as in order to carry out measurements within the broad range of flow and different slurry formulation every time it is necessary to readjust the equipment with the objective of ensuring proper measuring conditions.

In addition, these devices perform the viscosity measurement at a certain speed of medium motion, so the obtained results can be considered adequate only under certain conditions.

Volarovich viscosimeter is the most appropriate for the evaluation of rheological parameters of sand-lime mortars with different mobility (Kockal, 2016). But it has a rather complex structure and requires high accuracy of manufacturing and assembling of its components.

Having regard to the above, we have proposed a test method 
for determining the rheological properties of mortar mixtures with different mobility, which is the study of the interaction between a metal ball and the test solution.

An example of a study of the interation between a spherical body and the liquid is the Stokes problem of a slow static flowing round the ball, in which the main influence is created by pressure and friction forces, and inertial components are not taken into account. A real example, closest to such a movement, is a slow drop of the ball in very viscous liquid (Korobko B and Vasyliev, 2014), to which sand-lime mortar can also be relevant.

The solving of the Stokes problem, when the ball is flowed round by structural liquid, the rheological behavior of which is determined by Shvedov-Bingham's law (1), takes the following form:

- the normal stress pattern on the ball surface of radius $r$ at static relative motion of a ball and of the liquid at the velocity of $V_{0}$ is as follows

$p=-\frac{3}{2} \cdot \frac{V_{0} \cdot \eta}{r} \cdot \cos \varphi$,

where: $\varphi$ - is the central angle between the direction of the velocity vector $\vec{V}_{0}$ of flow and the normal to the concerned element of the spherical surface of the ball;

- shear stresses, caused only by viscosity and velocity gradients in the boundary layer, are spread on the surface of the ball in this way:

$\tau_{\eta}=\frac{3}{2} \cdot \frac{V_{0} \cdot \eta}{r} \cdot \sin \varphi$,

and with a glance to the critical shear stress of the environment $\tau_{0}(1)$

$\tau_{\eta}=\tau_{0}+\frac{3}{2} \cdot \frac{V_{0} \cdot \eta}{r} \cdot \sin \varphi$.

The forcing, which is a measure of the interaction between a ball and structural liquid, consists of two components

$F=F^{\prime}+F^{\prime \prime}$.

The components of the force $F$ are defined as the sum of projections of elementary efforts, caused by the availability of normal $p$ and shear $\tau$ stresses on the surface of the ball (Fig. 1):

$F^{\prime}=\int_{S} \tau \cdot \cos \left[90^{\circ}-\left(180^{\circ}-\varphi\right)\right] d S$,

$F^{\prime \prime}=\int_{S} p \cdot \cos \left(180^{\circ}-\varphi\right) d S$.

Formula (5) after the conversion of expressions for $F^{\prime}$ and $F^{\prime \prime}$ will be of the following form

$F=\int_{S} \tau \cdot \sin \varphi d S-\int_{S} p \cdot \cos \varphi d S$,

where: $d S$ - is the area of the ball's surface element,

$d S=2 \cdot \pi \cdot r^{2} \cdot \sin \varphi d \varphi$.

Having inserted the relations (2) and (4) into (6), we will get the formula for determining the resistance force $F$, which occurs when a ball of radius $r$ moves with respect to the mortar mix at a speed of $V_{0}$ :

$F=\int_{0}^{\pi}\left(\tau_{0}+\frac{3}{2} \cdot \frac{V_{0} \cdot \eta}{r} \cdot \sin \varphi\right) \cdot \sin \varphi \cdot 2 \cdot \pi \cdot r^{2} \cdot \sin \varphi d \varphi-$

$-\int_{0}^{\pi}\left(-\frac{3}{2} \cdot \frac{V_{0} \cdot \eta}{r} \cdot \cos \varphi\right) \cdot \cos \varphi \cdot 2 \cdot \pi \cdot r^{2} \cdot \sin \varphi d \varphi$,

$F=\pi^{2} \cdot r^{2} \cdot \tau_{0}+6 \cdot \pi \cdot V_{0} \cdot r \cdot \eta$.

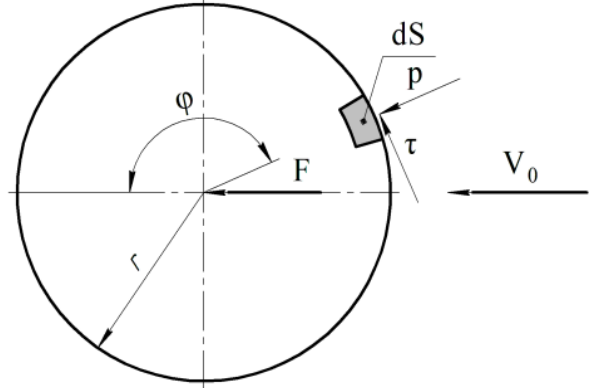

Fig. 1. The scheme of interaction between the ball and the flow of mortar mix

The first summand of the expression (7) is independent of the structural solution viscosity $\eta$ and the speed of ball's movement. It defines the component of the total force, which is necessary for shifting the ball towards the mortar mix, which creates static resistance to its motion, caused by the presence of $\tau_{0}$ stress. The second summand is determined by the traverse speed of the ball $\mathrm{V}_{0}$ relative to the mortar mix.

Analyzing the expression (7), we can come to the conclusion, that the determination of rheological parameters $\tau_{0}$ and $\eta$ of the mortar mix should be carried out in two stages.

At first it is necessary to define the force, at which there occurs the shift of the ball of radius $r$, which is in the mortar mix. This allows you to calculate the value of $\tau_{0}$, provided that at the beginning of the shift of the ball its speed through mortar mix becomes zero. Then the expression (7) will be as follows $F_{\tau}=\pi^{2} \cdot r^{2} \cdot \tau_{0}$, from which

$\tau_{0}=\frac{F_{\tau}}{\pi^{2} \cdot r^{2}}$.

The value $F_{\tau}$ is defined as the difference between the external force $\mathrm{F}_{0}$, at which the ball shear occurs, and the buoyancy force $\mathrm{F}_{\mathrm{A}}$.

Then there are measured the speed $V_{0}$ and the nature of ball's motion in the mortar mix under the action of constant external force $F$. After determination of the velocity $V_{0}$ and the stress $\tau_{0}$ on the basis of the expression (7) there is calculated the value of the plastic solution viscosity $\eta$

$\eta=\frac{F-\pi^{2} \cdot r^{2} \cdot \tau_{0}}{6 \cdot \pi \cdot V_{0} \cdot r}$.

With allowance for the buoyancy force, the calculating formulae (8) and (9) ultimately will be of the following form:

$\tau_{0}=\frac{F_{0}-\frac{4}{3} \cdot \pi \cdot r^{3} \cdot g \cdot \rho}{\pi^{2} \cdot r^{2}}$,

$\eta=\frac{\mathrm{F}-\frac{4}{3} \cdot \pi \cdot r^{3} \cdot g \cdot \rho-\pi^{2} \cdot r^{2} \cdot \tau_{0}}{6 \cdot \pi \cdot V_{0} \cdot r}$

where: $\rho$ - is the density of the test solution, $\mathrm{kg} / \mathrm{m} 3, \mathrm{~g}-$ is the gravitational acceleration, $\mathrm{m} / \mathrm{s} 2$.

In order to study the interaction between the ball and the test solution, we have used the apparatus (see Fig. 2), which consists of a steel ball 1 , rigidly connected with the steel rod 2 . At the rod there was located bearing plate 3 for the load of balance weights. At determining the initial shift of the ball, on the end of the rod there is placed a pointer against the corresponding scale mark, situated on the bracket 4 , which is connected to the blending hopper. When investigating the ball's motion pattern in the mortar mix on the bracket 4 , there is set a displacement pickup of inductive type 5 . 


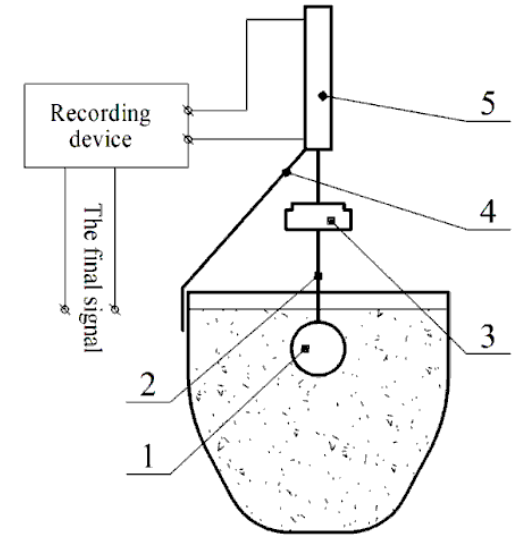

Fig. 2. The device for measuring rheological characteristics of the mortar mix

Measurements are performed in such a way. The ball 1 is submerged into the freshly-mixed sand-lime mortar with the proportion of ingredients 1: 3 in such a way, so that it would be completely covered with the mortar. Balance weights are consistently set on the bearing plate 3 in such a way, so that the difference in load between them would be no more than $5 \mathrm{~g}$. Keeping watch over the pointer, we fix the shear couple of the ball. The magnitude of applied force $F_{0}$, which corresponds to the beginning of shift, is defined as the sum of fare weight of a ball with a rod and the weight of balance weights, established on the bottom. The density of the mortar mix is determined by weighing the fixed volume of the mortar. Then the critical shear stress $\tau_{0}$ for the test solution is determined from the formula (10). The mobility of the mortar mix is measured by using the slump cone "StroyTsNIL". When measuring the ball's rate of descent in the test solution, the load weighing $2.5 \ldots 3$ times larger than $F_{0}$ is set on the bearing plate (this is necessary to ensure the sinking of the ball in the mortar mix). The mortar mix is stirred in the slurry blender. Immediately after the shut-down of the stirring the ball 1 is sunk in such a way, so that it would be completely covered by the mortar mix, and it is held in this position. The self-recording instrument is switched on and the ball is released.

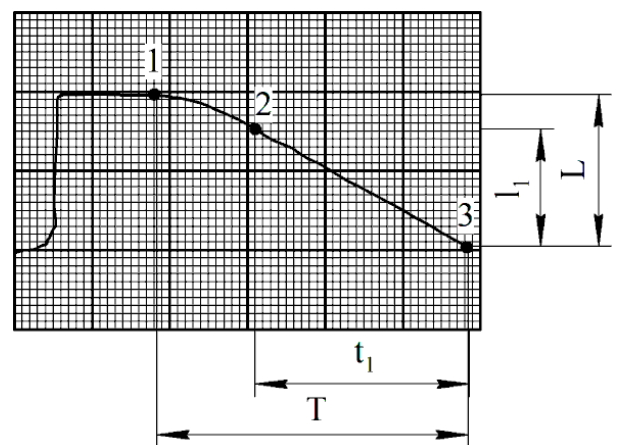

Fig. 3. The displacement diagram, showing the movement of the ball $60 \mathrm{~mm}$ in diameter in the sand-lime mortar with the composition 1:3 and with the medium workability of $8 \mathrm{~cm}$

In Fig. 3 there is given a displacement diagram, showing the movement of the ball $60 \mathrm{~mm}$ in diameter in the sand-lime mortar with the composition 1: 3 and with the medium workability of $8 \mathrm{~cm}$.

The chart shows that at a certain period of time the ball is moving at a constant speed (points 2-3), that is, the interaction process is stationary in nature. One cell of the diagram in the horizontal direction corresponds to $0.01 \mathrm{~s}$. The analysis of the diagram testifies that the full time of the ball's release $T$ is equal to 0.39 (segment $T=39 \mathrm{~mm}$ ), and the ball's stationary motion $t_{1}$ $=0.27 \mathrm{~s}$ (segment $t_{1}=27 \mathrm{~mm}$ ). The ball's depth of immersion is determined based on the results of the movement sensor calibration (in the diagram $L=323 \mathrm{~mm}, l_{1},=255 \mathrm{~mm}$ ).

The study of the received diagram allows to determine the velocity of the ball in the mortar mix at the steady motion area according to the formula $V_{0}=\frac{l_{1}}{t_{1}}$ and, using the relationship (11), to calculate the value of the plastic viscosity of the test solution $\eta$.

The criterion which determines the process of hydrodynamic interaction between a valve ball and the flow of mortar is Reynolds criterion, which characterizes the effect of medium viscosity forces while flowing round the ball. In estimating flow motion of viscous medium in cylindrical space Reynolds criterion is as follows:

$R e=\frac{V_{0} \cdot 2 \cdot r}{\eta} \cdot \rho$.

When investigating the medium motion, which has got structured viscosity, the generalized parameter of Reynolds ought to be determined in the following way:

$R e^{*}=R e \frac{6 \cdot \eta \cdot V_{0}}{6 \eta \eta \cdot V_{0}+\tau_{0} \cdot 2 \cdot r}$.

Using the above mentioned methods, we have carried out measurements by applying the sand-lime mortar with the composition $1: 3$ and with the medium mobility ranging from 8 to $12 \mathrm{~cm}$. The results of measurement are using a 40,50 and $60 \mathrm{~mm}$ diameter steel ball are given in the Tab. 1.

Tab 1. The measuring results of rheological characteristics of sand-lime mortar mixes with different mobility

\begin{tabular}{|c|c|c|c|c|c|c|c|c|}
\hline $\begin{array}{c}\text { Mobility, } \\
{[\mathrm{cm}]}\end{array}$ & $\begin{array}{c}\text { Density } \rho, \\
{\left[\mathrm{kg} / \mathrm{m}^{3}\right]}\end{array}$ & $\begin{array}{c}d, \\
{[\mathrm{~mm}]}\end{array}$ & $F_{0},[\mathrm{~N}]$ & $\begin{array}{c}\tau_{0}, \\
{[\mathrm{~Pa}]}\end{array}$ & $F,[\mathrm{~N}]$ & $\begin{array}{c}V_{0}, \\
{[\mathrm{~m} / \mathrm{s}]}\end{array}$ & $\begin{array}{c}\eta, \\
{[\mathrm{Pa} \cdot \mathrm{s}]}\end{array}$ & $R e^{*}$ \\
\hline \multirow{3}{*}{8} & \multirow{3}{*}{2100} & 60 & 7.75 & 610.22 & 24.07 & 0.94 & 30.53 & 3.21 \\
\cline { 3 - 9 } & & 50 & 5.10 & 608.43 & 15.30 & 0.74 & 29.20 & 2.14 \\
\cline { 3 - 9 } 10 & \multirow{3}{*}{2000} & 40 & 3.14 & 607.92 & 8.40 & 0.45 & 31.22 & 0.93 \\
\cline { 3 - 9 } & & 60 & 6.42 & 473.60 & 17.85 & 1.21 & 16.71 & 7.03 \\
\hline \multirow{3}{*}{12} & \multirow{3}{*}{1900} & 40 & 4.21 & 475.70 & 11.75 & 1.06 & 15.07 & 5.65 \\
\cline { 3 - 9 } & & 60 & 4.80 & 303.86 & 11.43 & 0.98 & 12.00 & 7.40 \\
\cline { 3 - 8 } & & 40 & 1.81 & 301.51 & 5.25 & 0.81 & 11.27 & 4.47 \\
\hline
\end{tabular}

For checking the proper use of analytic consistent patterns, the authors have sized up the actual magnitude of Reynolds parameter, which is the criterion of the process' temporal homogeneity. The calculation data of $R e^{*}$ according to the formula (13) for every dimension are listed in the table 1 . This parameter value in all dimensions does not exceed $R e^{*}=10$, what offers an opportunity to draw a conclusion about the accuracy of the developed methodology. 


\section{CONCLUSIONS}

The analysis of the obtained measured data and calculation data points out that in case of reduction in diameter of the ball, its zero-time shift in the mortar of certain mobility requires less efforts. When increasing the mobility of the mortar, the effort of zero-time shift of the ball of certain diameter decreases either.

Calculation data for each ball testify that the values of boundary traction of the shift and the coefficient of structured viscosity of the mortar of certain mobility are in narrow interval, that does not exceed systematic inaccuracy of measurements. That is why for the mortar of target slump these dimensional characteristics can be determined as arithmetical average of measured data for each ball. Moreover, in case of increase of mobility of the analyzed mortar, the values of boundary traction of the shift and the coefficient of structured viscosity decrease substantially. It makes it possible to confirm that rheological properties of building mortars depend only on their consistence.

The proposed method makes it possible to quickly determine the rheological properties of mortar mixes and other structural liquids, which follow Shvedov-Bingham's law, immediately prior to their conveyance by the mortar pump.

It will allow to predict energy costs during the work of equipment set for plastering: expenditures for mixing in the mixer tap, injection of grout by the mortar pump and transportation of mortars by pipeline. The design extent of power consumption will help to determine the necessary propulsive output as part of equipment set.

\section{REFERENCES}

1. Almeida Alessandra E. F. de S., Sichieri Eduvaldo P. (2007), Experimental study on polymer-modified mortars with silica fume applied to fix porcelain tile, Building and Environment, 42(7), 2645-2650.

2. Assaad Joseph J., Daou Yehia. (2014), Cementitious grouts with adapted rheological properties for injection by vacuum techniques, Cement and Concrete Research, 59, 43-54.

3. Binici Hanifi, Gemci Remzi, Kaplan Hasan. (2012), Physical and mechanical properties of mortars without cement, Construction and Building Materials, 28(1), 357-361.

4. Chen X., Wu S., Zhou J., Chen Y., Qin A. (2013), Effect of testing method and strain rate on stress-strain behavior of concrete, Journal of Materials in Civil Engineering, 25(11), 1752-1761.

5. Cuia Hongzhi, Liaoa Wenyu, Mia Xuming, Lob Tommy Y., Chenc Dazhu. (2015), Study on functional and mechanical properties of cement mortar with graphite-modified microencapsulated phasechange materials, Energy and Buildings, 105, 273-284.

6. González-Fonteboa B., Martínez-Abella F. (2008), Concretes with aggregates from demolition waste and silica fume. Materials and mechanical properties, Building and Environment, 43(4), 429-437.

7. Kheradmand Mohammad, Azenha Miguel, Aguiar Jose L.B. de, Krakowiak Konrad J. (2014), Thermal behavior of cement based plastering mortar containing hybrid microencapsulated phase change materials, Energy and Buildings, 84, 526-536.

8. Kim Kwan Ho, Cho Hee Chan, Ahn Ji Whan. (2012), Breakage of waste concrete for liberation using autogenous mill, Minerals Engineering, 35, 43-45.

9. Kockal Niyazi Ugur. (2016), Investigation about the effect of different fine aggregates on physical, mechanical and thermal properties of mortars, Construction and Building Materials, 124, 816-825.

10. Korobko B.O. (2016) Investigation of energy consumption in the course of plastering machine's work, Eastern-European Journal of Enterprise Technologies (Energy-saving technologies and equipment), 4, 8 (82), 4-11.

11. Korobko B.O., Vasyliev I.A. (2012) Laboratory test stand for the mortar pump loading (in Ukrainian), Collected works (branch of engineering, construction), Poltava, PoltNTU, 1 (31), 82-86.

12. Korobko B.O., Vasyliev I.A. (2014), Mortar ball valve efficiency research according to the law of the piston (in Ukrainian), Collected works (branch of engineering, construction), Poltava, PoltNTU, 1(40), 14-19.

13. Perrot A., Rangeard D., Picandet V., Mélinge Y. (2013), Hydromechanical properties of fresh cement pastes containing polycarboxylate superplasticizer, Cement and Concrete Research, 53, 221-228.

14. Reis J.M.L., Moreira D.C., Nunes L.C.S., Sphaier L.A. (2011), Experimental investigation of the mechanical properties of polymer mortars with nanoparticles, Materials science and engineering A-Structural materials properties microstructure and processing, 528(18), 6083-6085. 